\title{
1 Modulation of auditory responses by visual inputs in the mouse 2 auditory cortex
}

- Key words: Multisensory, noise correlations, suppression, Layer 2/3 -visual responses in auditory cortex, hyperpolarization

- Sudha Sharma* ${ }^{1}$, Hemant Kumar Srivastava* ${ }^{1}$ and Sharba Bandyopadhyay**1,2

${ }^{1}$ Advanced Technology Development Centre, IIT Kharagpur

*equal contribution Trust DBT India Alliance for Grant IA/I/11/2500270 and IIT Kharagpur for SRIC Challenge Grant SGIGC-2015/DMN and MHRD SSLS scheme for funds 


\section{Abstract}

So far, our understanding on the role of the auditory cortex (ACX) in processing visual information has been limited to infragranular layers of the ACX, which have been shown to respond to visual stimulation.

22 Here, we investigate the neurons in supragranular layers of the mouse ACX using 2-photon calcium

23 imaging. Contrary to previous reports, here we show that more than $20 \%$ of responding neurons in layer $2 / 3$ of the ACX respond to full-field visual stimulation. These responses occur by both excitation and hyperpolarization. The primary $\mathrm{ACX}$ (A1) has a greater proportion of visual responses by hyperpolarization compared to excitation likely driven by inhibitory neurons of the infragranular layers

27 of the ACX rather than local layer $2 / 3$ inhibitory neurons. Further, we found that more than $60 \%$ of neurons in the layer $2 / 3$ of $A 1$ are multisensory in nature. We also show the presence of multisensory neurons in close proximity to exclusive auditory neurons and that there is a reduction in the noise correlations of the recorded neurons during multisensory presentation. This is evidence in favour of deep and intricate visual influence over auditory processing. The results have strong implications for

32 decoding visual influences over the early auditory cortical regions.

34 To understand, what features of our visual world are processed in the auditory cortex (ACX), understanding response properties of auditory cortical neurons to visual stimuli is important. Here, we show the presence of visual and multisensory responses in the supragranular layers of the ACX.

37 Hyperpolarization to visual stimulation is more commonly observed in the primary ACX. Multisensory stimulation results in suppression of responses compared to unisensory stimulation and an overall decrease in noise correlation in the primary ACX. The close-knit architecture of these neurons with auditory specific neurons suggests the influence of non-auditory stimuli on the auditory processing. 


\section{Introduction}

42

43

44

45

46

47

Multisensory integration allows seamless assimilation of information from different sensory modalities to better perceive the environment. Cross-modal interaction of incoming sensory information is important since each sensory system is capable of encoding the same stimulus in a uniquely advantageous way(Maddox et al., 2015; Bizley et al., 2016). So far, most of our understanding of visual influence on the auditory pathway is with respect to sound source localization by subcortical regions(Werner-Reiss et al., 2003; Bulkin and Groh, 2012) and little is known of it in the ACX(Kayser et al., 2010; Atilgan et al., 2018; Morrill and Hasenstaub, 2018). However, other functions like communication and speech perception are higher cognitive functions(Ghazanfar, 2005, 2009; Skipper et al., 2007; Koelewijn et al., 2010; Romanski and Hwang, 2012) and a cross-talk between visual and auditory cortices is necessary for enhanced speech perception. Therefore, a basic understanding of this architecture in the animal models can help us decipher the framework over which these complex processes are built. Several studies have shown the cross-modal nature of sensory cortices(Ghazanfar and Schroeder, 2006). However, there is growing number of studies that deal with auditory influence over visual cortex(Ibrahim et al., 2016a; Murray et al., 2016; Meijer et al., 2017; Deneux et al., 2018; Knöpfel et al., 2019) rather than vice versa(Bizley and King, 2008a; King and Walker, 2012; Atilgan et al., 2018; Morrill and Hasenstaub, 2018). This is likely due to the easy accessibility of visual cortex with advanced tools like 2-photon imaging, which has not been used to explore the role of the mouse ACX in multisensory processing yet.

Although there is enough evidence of direct projections from the primary visual cortex to auditory areas in rodents other than mice(Budinger et al., 2006; Bizley et al., 2007; Campi et al., 2010), they are much less dense than those in the reverse direction(Oh et al., 2014; Ibrahim et al., 2016a). The reason for this disparity is unknown. Many physiological studies of visual influence over the A1 in ferrets and in 
64 primates have shown to affect the phase of oscillatory activity in the ACX, causing amplification of the

65 response to an auditory stimulus(Ibrahim et al., 2016a; Atilgan et al., 2018) in presence of an audiovisual stimulus. This amplification leads to decreased response latencies and enhanced responses in a

67 noisy background(Wang et al., 2008). Other studies also report spiking activity in the ACX by visual

68 stimuli, but also that this spiking is limited to infragranular layers(Bizley et al., 2007; Morrill and Hasenstaub, 2018) and absent in supragranular layers. These studies have resorted to electrophysiology, which lacks spatial resolution. This has led to the assumption that the visual responses in the ACX either

71 are the result of local field potential modulation from the surrounding visual cortex or are only present

72 in the deeper layers of the auditory.

73 Using two-photon calcium imaging with GCaMP6s in awake mouse, we probed layer 2/3 (supragranular)

74 of the ACX with auditory, visual, and audio-visual (or multisensory) stimuli. In addition to responses to

75 auditory stimulation (broadband noise), we found robust Ca2+ based responses to visual stimulation in

76 layer $2 / 3$ of primary as well as secondary ACX. Most of the neurons show suppressive responses to

77 multisensory stimulus as compared to their unimodal counterparts. This study also reveals neurons

78 having different response strengths towards the auditory, visual and multisensory stimuli. Two-photon

79 calcium imaging also reveals the close-knit spatial architecture of the auditory and multisensory

80 neurons, as well as an effect of multisensory stimuli on the noise correlations in the primary as well as

81 secondary auditory areas. This paper is an attempt towards understanding the complex nature of

82 multisensory processing in the ACX by studying the inherent capabilities of these neurons to perform

83 multisensory processing without undergoing any plasticity due to multisensory experiences. 
All procedures were in accordance with protocols approved by the Indian Institute of Technology Kharagpur, Institutional Animal Care and Use Committee and guidelines of the National Institutes of Health. Mice were acquired from Jackson Laboratories (PV-Cre [JAX 008069], SOM-IRES-Cre [JAX 013044], ROSA LSL-tdTomato [JAX 007908]) housed in a room with a reversed light cycle. Experiments were performed during the dark period.

Surgery: Adult mice (>30 days old, male and female) were anesthetized with isoflurane ( $5 \%$ induction and $1 \%$ maintenance), injected with dexamethasone $(2 \mathrm{mg} / \mathrm{kg}$ body weight) intraperitoneally and placed on a stereotaxic frame. Body temperature of the animal was maintained at $37{ }^{\circ} \mathrm{C}$ throughout the procedure using a heating pad. Incision was made to expose the skull. A craniotomy ( $3 \mathrm{~mm}$ diameter) was done to expose the ACX. Virus (AAV.Syn.GCaMP6s.WPRE.SV40) was loaded into a glass micropipette mounted on a Nanoject II attached to a micromanipulator and injected at a speed of $20 \mathrm{~nL}$ per min at several places exposed by the craniotomy in PV-tdTomato and SOM-tdTomato transgenic animals. The craniotomy was covered with a glass coverslip(Goldey et al., 2014). Mice were left in their home cage to 101 recover and virus to integrate and express for 2 weeks. Experiments were conducted 15-20 days after 102 viral injection. recording locations. Mice were deeply anesthetized with isoflurane followed by transcardial perfusion

105 with $0.1 \mathrm{M}$ phosphate-buffered saline followed by $4 \%$ paraformaldehyde. Brains were harvested and 106 after a post-fixation period of 8-10 hours in $4^{\circ} \mathrm{C}, 100$ um thick sections were cut using a vibratome (Leica 107 VT1000S) and images were taken in a fluorescence microscope (Leica DM2500). 
Auditory and Visual stimulation: Auditory stimuli were presented to the ear contralateral to the recording hemisphere inside a light-proof chamber, $10 \mathrm{~cm}$ away from the right ear (contralateral) of the mouse. Stimulus was generated through custom-written software in MATLAB (Mathworks), passed

111 through TDT RZ6 multifunctional processor. Acoustic calibrations were performed using microphone

112 (Brüel \& Kjær, Denmark) and showed a typical flat (+/- $7 \mathrm{~dB}$ ) calibration curve from 4-60 kHz. All mice 113 were first presented with pure tone ( $T$ stimulus, 5 repetitions each, $6-48 \mathrm{kHz}, 1 / 2$ octaves apart, $50 \mathrm{~ms}$ 114 duration with $5 \mathrm{~ms}$ rise and fall, 5 s inter stimulus interval at $~ 65-75 \mathrm{~dB}$ SPL) in order to identify the 115 tonotopic axis. For comparison of unisensory vs. multisensory responses, we chose to play white noise 116 (WN, bandwidth $6-48 \mathrm{kHz}, 10$ repetition) instead of pure tones as the auditory stimulus (to be later 117 paired with visual LED flash for multisensory stimulation) in order to avoid the influence of frequency 118 selectivity of individual neurons. For visual stimulation, a white LED (5 mm Round White LED(T-1 3/4)) 119 kept $10 \mathrm{~cm}$ away from the eye of the animal, close to the source of sound, was used. Full-field 120 illumination to the contralateral eye was provided and the intensity (1-160 lux, Model: LX1010b, Digital 121 Lux Meter, 0 to 20000 lux) was controlled through the NI DAQ card, using MATLAB routines. LED blink 122 stimulus was presented for $10 \mathrm{~ms}$ ( $V$ stimulus) with an interstimulus interval of $5 \mathrm{~s}$ in order for the cones 123 in the retina to recover. Audio-visual stimulation (or Multisensory, $M$ stimulus) comprised of 124 simultaneous presentation of the $A$ and $V$ stimuli $(M)$, as above. 10 repetitions of the $M$ stimuli were 125 presented. Widefield calcium imaging: Images were acquired using a 14-bit CCD CoolSNAP HQ2 CCD camera

127 (Photometrics). Images of surface vasculature were acquired using blue LED illumination (470 nm), and 128 wide-field GCaMP signals were recorded (36 ms frame period) using blue illumination (470 $\mathrm{nm}$ ). 129 Fluorescence signals were acquired at $4 x$ binning of 1040x1392 (260X348) pixels using a $4 x$ 130 objective(Olympus). Each trial consisted of 50 baseline frames and 50 frames of response. A 500 ms 
131 sound stimulus $(60-30 \mathrm{~dB}$ SPL pure tone with a frequency in the range $4-48 \mathrm{kHz}, 30$ trials for each

132 frequency) was presented starting at the $51^{\text {st }}$ frame. The inter-trial interval was 5-7 s. Images during the

133 response period ( $0.5-2 \mathrm{~s}$ from the sound onset) were averaged, and the average image during the

134 baseline was subtracted from it. This difference was divided by the average image during the baseline.

$135 \quad$ 200-300 ms after stimulus start was considered as a response window.

136 2-photon calcium imaging: Two-photon calcium imaging was performed using a commercially available

137 2-photon microscopy system (Prairie View Technologies). The microscope was controlled by Prairie View

138 software (Prairie View Technologies). For imaging, the excitation beam of wavelength 860-920nm

139 generated from Insight laser (Spectra-Physics, USA) was directed over the glass-covered brain surface in

140 layer 2/3 (180-250um depth). The laser was delivered through 20X/0.8 NA water immersion objective

141 (2mm WD, Olympus). The laser power was adjusted from $50 \mathrm{~mW}$ to $80 \mathrm{~mW}$ depending upon the

142 condition of the specimen. Frames in the region of interests $(\sim 150 \mu \mathrm{m} \times 200 \mu \mathrm{m}$ with $1.16 \mu \mathrm{m}$ pixel size)

143 were imaged at $\sim 4-6 \mathrm{~Hz}(160-250 \mathrm{~ms}$ frame period, 1.2-4 $\mu$ s dwell time) with the stimulus presented at

144 10th frame in a sequence of 20 frames per stimulus. Cross-correlation-based image alignment was used

145 for motion correction in XY plane. Center of cells was marked manually and rings were drawn within of

$1465 \mu \mathrm{m}$ radius around the point of selection. Luminance $\left(F_{1}\right)$ of all the pixels within the rings was the mean

147 of all pixel values within the marked region. Baseline fluorescence was subtracted to calculate change in

148 fluorescence and normalized by the baseline fluorescence to compute $\mathrm{dF} / \mathrm{F}$. All other analysis was done

149 on these $\mathrm{dF} / \mathrm{F}$ values.

Data Analysis:

151 Widefield Imaging data analysis: For creating a tonotopic map, we used $5^{\text {th }}$ to $10^{\text {th }}$ frame (180-360 ms)

152 after the stimulus as the response frames. Mean along the 30 iterations was calculated for each of the 153 response frames. Using these mean responses frames, we computed the $\mathrm{dF} / \mathrm{F}$ by subtracting the mean 
of baseline frames and then dividing by it. The $\mathrm{dF} / \mathrm{F}$ was smoothed by a gaussian filter with standard deviation of 2. Next, this $\mathrm{dF} / \mathrm{F}$ matrix was normalized by the maximum to bring the range between -1 and 1. To this matrix, we applied a threshold above which all values were set to 1 and pixel values below threshold were set to zero. The threshold used was generally $65-75 \%$ of the maximum pixel value. The three image matrices, one each for low, middle and high frequencies were overlaid on each other to illustrate the tonotopic axis (Fig. 1, top row, right). the ACX in five animals injected with AAV.Syn.GCaMP6s in the ACX. All animals were exposed to low, middle and high frequencies for identifying the A1 tonotopic axis and to mark A1 within the craniotomy and an LED flash for demarcating the visual cortex boundary medial to AuD based on wide field calcium responses to LED. Based on the responses obtained, the imaging region was divided into four sub regions, secondary visual cortex (V2) which showed highest response activity to visual stimulus, A1 containing the tonotopic axis, AuD is the region between $\mathrm{V} 2$ and $\mathrm{A} 1$, and $\mathrm{AuV}$ to be below the marked A1 boundary. All recorded ROIs were then assigned a region, depending on their location. For this study, we excluded ROIs from the $\mathrm{V} 2$ region from our analysis.

169 Calculation of significant response and its reliability: To identify a significant response to a given 170 stimulus, we compared the mean baseline activity of three frames before the stimulation with the mean 171 response activity of three frames after stimulation in all iterations using one-tailed paired t-test. We 172 considered $p$ value less than $5 \%$ for the response by excitation and less than $10 \%$ for the response by 173 hyperpolarization as our criterion for response. We also calculated the reliability of responses by 174 comparing the mean signal of the response frames in iteration with the mean of baseline frames. If this 175 value was greater than 1.5 standard deviation for excitation (and 1 standard deviation for hyperpolarization) of the baseline signal, it was considered as a response in that iteration. Reliability was 
calculated as the ratio of number of responding iterations and total iterations. Keeping the standard

178 deviation to calculate reliability and the criterion for significance same for both type of responses, we

179 were unable to get the hyperpolarization responses because the calcium indicators cannot effectively capture hyperpolarization(Chamberland et al., 2017).

Classification of neurons: We divided the neurons into different classes based on the stimulus they responded to. Neurons that responded only to visual stimulus are $V 0$, neurons that responded only to auditory stimulus are $A O$, neurons that responded only to multisensory stimulus are $M O$. Neurons that responded only to auditory stimulus but showed significant changes in response on presentation of multisensory stimulus are multisensory neurons type $M A$, neurons that responded only to visual stimulus but showed significant change in response on presentation of multisensory stimulus are multisensory neurons type $M V$. Neurons that responded to auditory stimulus and visual stimulus irrespective of response to multisensory stimulus are multisensory neurons type MAV.

\begin{tabular}{|c|c|c|c|}
\hline Cell types & V & A & MS \\
\hline V0 & 1 & 0 & 1 \\
\hline MV & 1 & 0 & $1^{*}$ \\
\hline M0 & 0 & 0 & 1 \\
\hline MAV & 1 & 1 & $0 / 1$ \\
\hline A0 & 0 & 1 & 1 \\
\hline MA & 0 & 1 & $1^{*}$ \\
\hline
\end{tabular}

a) Comparisons of reliabilities For comparison of reliabilities for a given population between different stimuli, bootstrap method (Efron and Tibshirani, 1993) was performed to obtain confidence intervals on the reliabilities. Bootstrap populations $(n=1000)$ were created by randomly sampling from reliabilities with replacement from the original dataset. We obtained 1000 reliability values from the population of 
1000 bootstrap sets. The mean reliabilities from two different conditions were considered to be

197 significantly different if the $99 \%$ confidence intervals of the two did not overlap with each other and accordingly for one-sided comparisons.

b) Spatial organization of neurons w.r.t type AO neurons: Distance between type $A 0$ and its nearest type $x$ (where, $x=V 0 / M V / M A / M A V / A 0)$ was obtained. These distances were averaged to obtain the mean distance (raw) between type A0 and type $\mathrm{x}$ neurons. Bootstrap population ( $n=1000)$ was created by randomly sampling with replacement (200 times) from these distances and averaged to obtain the confidence intervals from the mean values for comparison between populations. The raw mean distances from two different pairs were considered significantly different if the $95 \%$ confidence intervals

207 five cells to be present within 50um region. 


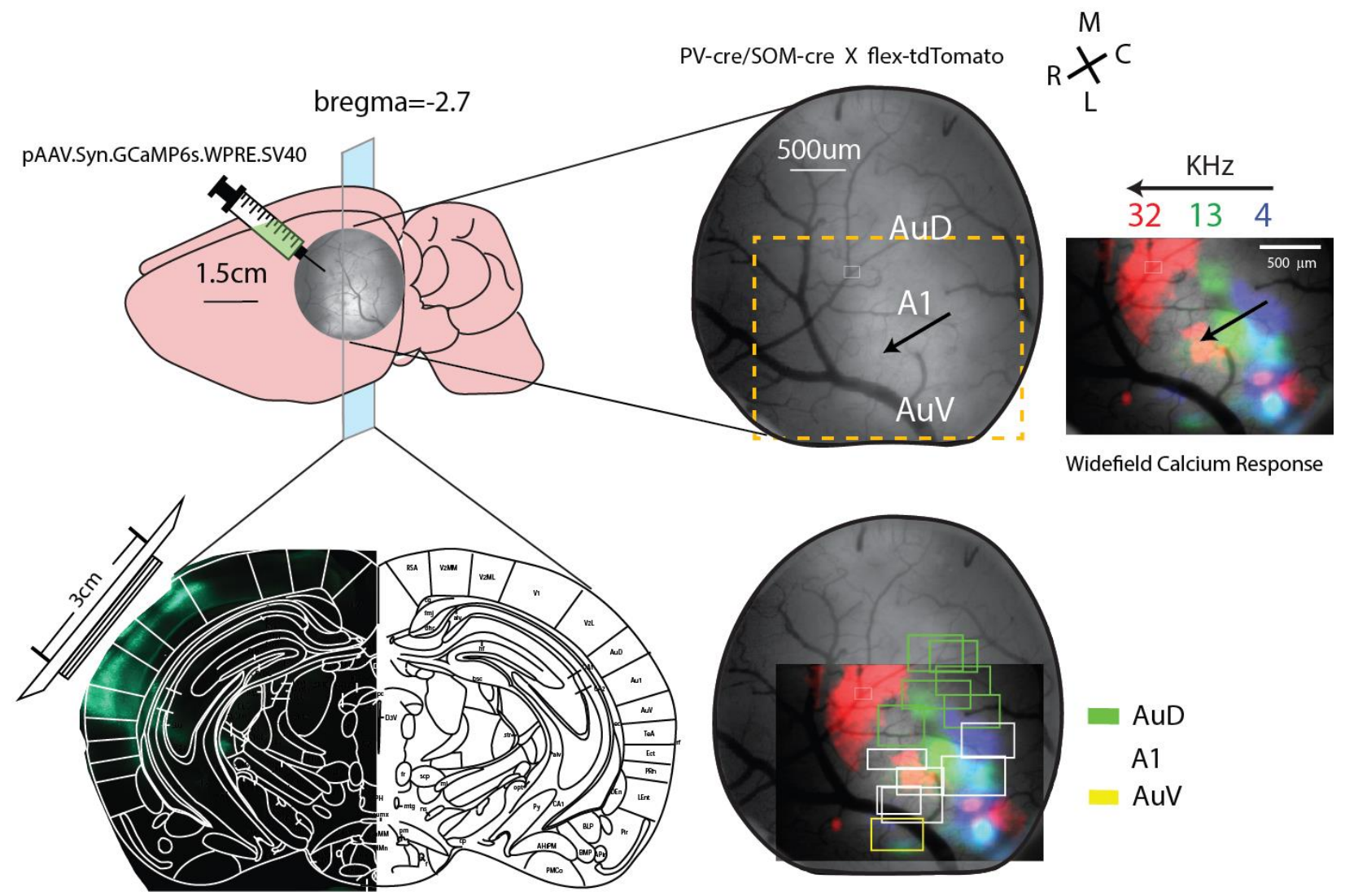




\section{Cross-modal influence is more prevalent in neurons of the ACX than their unisensory nature}

2-photon $\mathrm{Ca}^{+2}$ imaging was performed in layer $2 / 3$ of awake mice ( 3 animals (SOMxtd-tomato) and 2 animal (PARVxtd-tomato)) after implanting a cranial window with injections of AAV-GCaMP6s targeted at layer $2 / 3$ in multiple regions of the ACX. Using tonotopic axis as reference, ROIs were classified to belong to a particular auditory field (Fig. 1, Methods). For unisensory stimuli either Noise (50ms width, auditory) or a white LED flash (10 ms width, visual) was used. For multisensory stimulus the LED flash and noise were presented together with the same starting time and durations as mentioned above. Data from multiple ROIs in the ACX were collected over days. Each responding neuron was categorized into one of the six categories based on its response to different stimuli and region it belonged to as shown in Table 1 (Methods, Classification of Neurons).

Contrary to previous reports(Morrill and Hasenstaub, 2018), layer $2 / 3$ of all regions of the ACX was found to have neurons responding to visual stimulus, including neurons that responded only to visual stimulus (type V0) and not to auditory stimulus (Fig. 2A, Top row(left), Table 1). AuD and AuV had a greater proportion of $\mathrm{V} 0$ type neurons compared to A1 (AuD (4.1\%, 55/1335), A1(1.2\%, 32/2489), AuV (5.2\%, 58/1095)) (see Fig. 2B, Left and Table 1) while the greatest proportion of type A0 neurons were present in $\operatorname{A} 1$ (AuD (16.1\%, 215/1335), A1(33\%,842/2489), AuV (15.2\%, 167/1095) (Fig. 2B, middle). Multisensory neurons were prevalent in all auditory regions. However, there was some specificity observed with respect to the subtype of multisensory neurons and the region involved. $M V$ type neurons were maximum in $\operatorname{AuD}(19.2 \%, 257 / 1335)$, followed by AuV $(10.8 \%, 119 / 1095)$ and A1(6.4\%, 160/2489) (Fig. 2B, right), while MA type neurons were present with greatest proportion in A1 (34\%, 847/2489) and less in AuD (27.8\%, 372/1335) and even lesser in AuV (16.9\%, 186/1095) (Fig. 2B). MAV type neurons were mostly present in $\operatorname{AuV}(27.3 \%, 299 / 1095)$ with highest proportion followed by A1 (14.4\%, 359/2489) and $\operatorname{AuD}(8.0 \%, 108 / 1335)$. Apart from these, M0 formed a significant proportion of 
251

252

253

254

255

256

257

258

259

260

261

262

263

264

265

266

267

268

269

270

271

272

273

274

population responsive exclusively to multisensory stimulus and not responsive to the presentation of individual unisensory stimulus in all three regions $(A u D=24.5 \%, 328 / 1335, A 1=10.0 \%, 249 / 2489$, $\mathrm{AuV}=24.2 \%, 266 / 1095)$. Overall, more than $60 \%$ of cells in all three regions were multisensory in nature either responsive to only multisensory stimulus (type MO) or to both unisensory stimuli (type MAV) or undergoing cross modal modulation (type MA and MV). To discern the role of PV+ and SOM+ inhibitory interneurons in multisensory processing, we looked at the distribution of these neurons into different categories. We did not observe anything unusual that could point to their particular role. In fact, the distribution of $\mathrm{PV}+$ and $\mathrm{SOM}+$ neurons into the categories looked similar in proportions to the one in Fig. 2B. We did not explore their function further.

We also looked at the mean reliability of response of the six classified neuron types to different stimuli. Type $\mathrm{AO}$ and type MA neurons in A1 have high response reliability for auditory stimulus and low for visual stimulus (one-sided comparison of mean reliabilities based on bootstrap confidence intervals, $A 0(A)=0.63[0.62,0.65], A O(V)=0.22[0.21,0.23], M A(A)=0.56[0.55,0.58], M A(V)=0.22[0.21,0.23])$. While type V0 and type MV neurons have higher response reliability for visual stimulus and low for auditory stimulus $(\mathrm{VO}(\mathrm{V})=0.46[0.41,0.55], \mathrm{V} 0(\mathrm{~A})=0.28[0.26,0.39], \mathrm{MV}(\mathrm{V})=0.53[0.5,0.57], \mathrm{MV}(\mathrm{A})=0.27[0.24,0.3]$, Fig. 2C). Type AO and type $V 0$ neurons show similar response reliabilities for multisensory stimulus as that to auditory and visual stimulus respectively $(A 0(A)=0.63[0.62,0.65], A 0(M)=0.60[0.59,0.62]$, $\mathrm{VO}(\mathrm{V})=0.46[0.41,0.55], \mathrm{V} 0(\mathrm{M})=0.49[0.43,0.55]$, Fig. $2 \mathrm{C})$. However, type MV and type MA neurons shows significant reduction in reliability to multisensory stimulation compared to visual and auditory stimulation respectively $(\mathrm{MV}(\mathrm{V})=0.53[0.5,0.57], \quad \mathrm{MV}(\mathrm{M})=0.26[0.23,0.29], \quad \mathrm{AA}(\mathrm{A})=0.56[0.55,0.58]$, $\operatorname{MA}(M)=0.35[0.33,0.37])$. This is likely due to suppression of responses to multisensory stimulation which will be discussed later in the article. Type MO neurons have higher response reliabilities for multisensory stimulation while type MAV neurons have high response reliabilities for all three stimuli $(\mathrm{M0}=0.24(\mathrm{~V}), 0.29(\mathrm{~A}), 0.48(\mathrm{M}), \mathrm{MAV}=0.52(\mathrm{~V}), 0.61(\mathrm{~A}), 0.48(\mathrm{M}))$. AuD and $\mathrm{AuV}$ also follow the same trend 
275 (Fig.2C). Responses for auditory stimulus in type A0 population were found to be more reliable in A1

276 compared to AuD and AuV (AuD=0.54 [0.51, 0.57], A1=0.63[0.62, 0.65], AuV=0.51[0.48, 0.54]). The

277 distribution of response reliabilities is in agreement with our neuron classification scheme and shows

278 the difference between different populations.

Table 1. Number of responding neurons in each category in different areas. S, suppression; E,

\begin{tabular}{|l|l|l|l|l|l|l|l|}
\hline & V0 & MV(S,E) & MAV(S,E) & A0 & MA(S,E) & MO(S,E) & TOTAL \\
\hline AUD & 55 & $257(195,62)$ & $108(17,8)$ & 215 & $372(335,37)$ & $328(23,305)$ & 1335 \\
\hline A1 & 32 & $160(156,4)$ & $359(73,5)$ & 842 & $847(830,17)$ & $249(65,184)$ & 2489 \\
\hline AUV & 58 & $119(107,12)$ & $299(26,11)$ & 167 & $186(173,13)$ & $266(8,258)$ & 1095 \\
\hline TOTAL & 145 & $536(458,78)$ & $766(116,24)$ & 1224 & $1405(1338,67)$ & $843(96,747)$ & 4919 \\
\hline
\end{tabular}

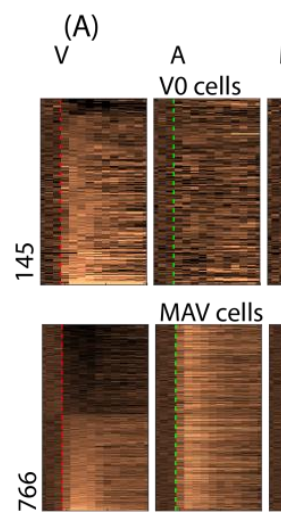

(B)

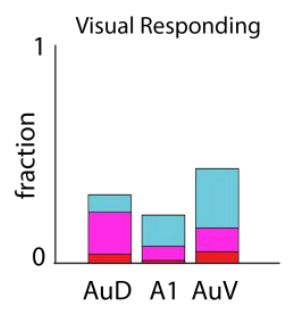

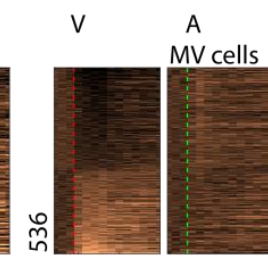
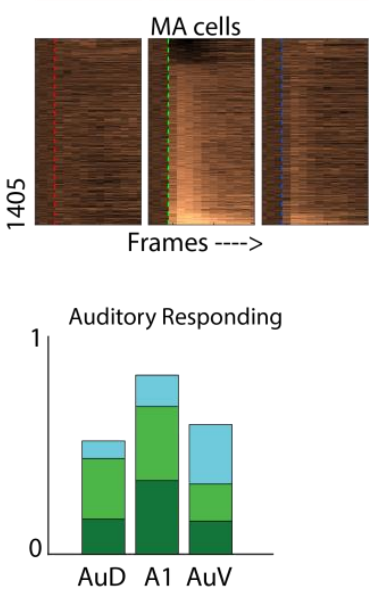

$-$
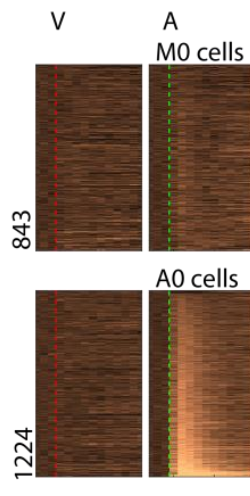

A0 cells

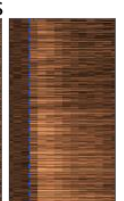

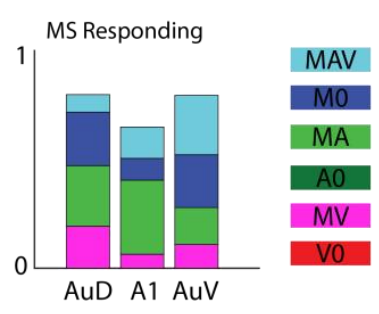

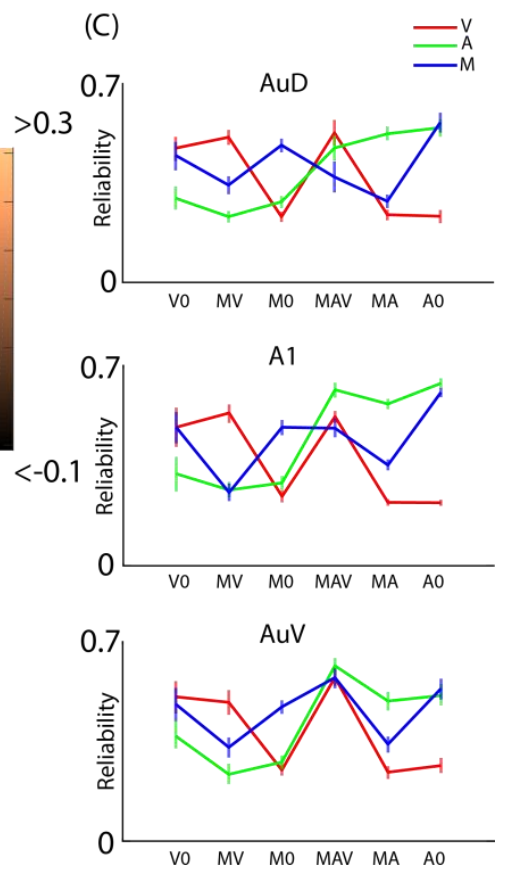


Figure 2. Classification, proportion and reliabilities of all cell types in different regions of the ACX. A,

284 Top row(left), type V0 cells with their mean $\mathrm{df} / \mathrm{f}$ values for auditory(A), visual(V) and multisensory(M) stimulation over total recorded frames. Red, Green and Blue lines on the images shows stimulation frame. Same scheme is repeated for type MV, MO cells in the top row and MAV, MA and AO cells in the bottom row. B, Proportion of neuron types, indicated by colour, as a fraction of total responding neurons in a region, Red: type V0 neurons, Pink: type MV neurons, Dark green: type A0 neurons, Light green: type MA neurons, Blue: type MO neurons, Cyan: type MAV neurons. C, Mean reliability values with confidence intervals obtained from bootstrap analysis of each type of population for region $A u D$ (top), $A 1$ (middle), $A u V($ bottom). Green: mean reliabilities on auditory stimulation, red: mean reliabilities on visual stimulation, blue: mean reliabilities on multisensory stimulation. neurons in the $A C X$, we looked at the local spatial organization of the primarily auditory type $A 0$ neurons and multisensory neurons with respect to each other in the primary and secondary auditory fields. We chose type A0 neurons because they are specific to auditory stimulation against all other, grouped in neurons in layer $2 / 3$ of all auditory regions to be similar, we compared the mean nearest neighbour distances between type $\mathrm{A} 0$ neurons and all other types, within and between auditory regions (Fig. 3B, Table 2). All comparisons were made based on the confidence intervals from the bootstrapped mean nearest neighbour distances (Methods, Table 2). We found that type A0 neuron is more likely to have 
by type $M A$ (AuD=39.53um, $A 1=29.48 u m, A u V=45.69 u m$ ) and type $M A V$ in close proximity

$307(A u D=53.21 u m, A 1=39.64 u m, A u V=46.36)$ in all three regions (Fig. 3B, Table 2). Type M0 neurons are closer to type $A 0$ neurons in $A u D$ and $A u V$ while they lie farther away in $A 1$ (AuD=40.59um, $A 1=83.77$ um, AuV=37.29um, Fig. 3B). Type V0 lie furthest away in A1 while they are relatively closer in AuD and even closer in AuV (AuD=102.00um, A1=132.93um, AuV=89.60um). Type MV neurons lie significantly closer to $A 0$ neurons than are $V 0$ neurons ( $A u D=81.55 u m, A 1=97.79 u m, A u V=68.04 u m)$.

312 We further looked at the probability of each type of neuron within 50um radius around type A0 neuron.

313 If most of the neurons in a 50um radius around a type $A 0$ neuron are of type $A 0$, probability of its 314 occurrence would be high, leading to auditory dominance in this region, and, if most of the neurons are 315 of type M, there would be multisensory dominance (Fig. 3C). We looked at contributions made by each 316 population type to the probability of type $M$ individually as well (Figure. 3C, Colour coded). All 317 comparisons were made on the mean probability values with $95 \%$ confidence intervals from the 318 bootstrapped data (Table 3). The mean probability value of type $M$ neuron around a type A0 neuron in 319 A1 is 0.48 (Fig 3C, Table 3). Hence, within a radius of 50um around a type A0 neuron $50 \%$ of cells on 320 average were found to be of type $\mathrm{M}$ while the other $50 \%$ are of type $\mathrm{A} 0$. While AuD and AuV are 321 multisensory dominant regions (probability in $\mathrm{AuD}=0.65, \mathrm{AuV}=0.75$ ). If we look at the contribution of 322 individual neurons types to the overall proabability value, type $\mathrm{V} 0(\mathrm{AuD}=0.03, \mathrm{~A} 1=0.0, \mathrm{AuV}=0.05)$ and 323 type $\mathrm{MV}(\mathrm{AuD}=0.05, \mathrm{~A} 1=0.01, \mathrm{AuV}=0.05)$ neuron are found very rarely within 50um in AuD and AuV 324 while being completely absent in A1. Type MA makes a higher contribution (AuD=0.21, $A 1=0.25$, $325 \mathrm{AuV}=0.16)$, followed by $\mathrm{MAV}(\mathrm{AuD}=0.1, \mathrm{~A} 1=0.15, \mathrm{AuV}=0.19)$ in all three regions. Another difference lies 326 in the type M0 population which has the highest contribution in AuD and AuV but not so much in A1 $327(\mathrm{AuD}=0.24, \mathrm{~A} 1=0.05, \mathrm{AuV}=0.26)$ 
From these observations, we propose presence of multisensory processing hub in auditory cortical areas at the center of which is a type AO neuron surrounded by MA and MAV in close proximity. Presence of type MAV neurons in close proximity of type $A 0$ neurons needs special mention as it reflects a direct visual influence over auditory processing. Probability of occurrence of different cell types within a given distance from type A0 neurons in $\mathrm{A} 1$ (Fig. 3D, middle) shows a decrease in probability of occurrence of type $\mathrm{A} 0$ neurons around the same type (shown in dark green) and an increase in the type $\mathrm{M}$ neurons with distance (shown in black) in all three regions. Probability of occurrence of type $M$ neurons remains high along with distance observed from type $A 0$ neurons in secondary areas, while in $A 1$, probability of occurrence of type $A 0$ is high in the nearby regions and reverses after 150 um(Fig. 3D, middle).

Table 2. Mean nearest neighbour distance of a cell type from type A0 neuron with $95 \%$ confidence

intervals with lower and upper bounds.

\begin{tabular}{|l|l|l|l|}
\hline & AuD (mean [L, U])(in um) & A1 (mean [L, U]) (in um) & AuV (mean [L, U]) (in um) \\
\hline V0 & $102.00[92.19,111.58]$ & $132.93[121.37,145.00]$ & $89.60[78.10,101.03]$ \\
\hline MA & $39.53[35.95,43.19]$ & $29.48[26.58,32.36]$ & $45.69[40.98,50.82]$ \\
\hline MAV & $53.21[48.11,58.63]$ & $39.64[35.58,44.19]$ & $46.36[41.26,51.27]$ \\
\hline MV & $81.55[74.79,87.85]$ & $97.79[89.64,106.15]$ & $68.04[62.65,73.63]$ \\
\hline M0 & $40.59[36.62,45.09]$ & $83.77[74.22,93.64]$ & $37.29[32.98,42.44]$ \\
\hline A0 & $34.86[30.21,39.49]$ & $21.03[18.83,23.61]$ & $31.05[28.13,34.39]$ \\
\hline
\end{tabular}




\begin{tabular}{|l|l|l|l|}
\hline & AuD (mean [L, U]) & A1 (mean [L, U]) & AuV (mean [L, U]) \\
\hline M & $0.65[0.61,0.69]$ & $0.48[0.44,0.51]$ & $0.75[0.72,0.77]$ \\
\hline V0 & $0.03[0.02,0.04]$ & $0.00[0.00,0.00]$ & $0.06[0.04,0.06]$ \\
\hline MA & $0.21[0.18,0.24]$ & $0.26[0.22,0.28]$ & $0.16[0.13,0.19]$ \\
\hline MAV & $0.10[0.08,0.12]$ & $0.15[0.13,0.17]$ & $0.20[0.16,0.23]$ \\
\hline MV & $0.06[0.04,0.07]$ & $0.02[0.01,0.02]$ & $0.06[0.04,0.07]$ \\
\hline M0 & $0.24[0.21,0.27]$ & $0.05[0.03,0.06]$ & $0.27[0.23,0.29]$ \\
\hline A0 & $0.35[0.30,0.38]$ & $0.52[0.48,0.55]$ & $0.25[0.22,0.27]$ \\
\hline
\end{tabular}

(A)

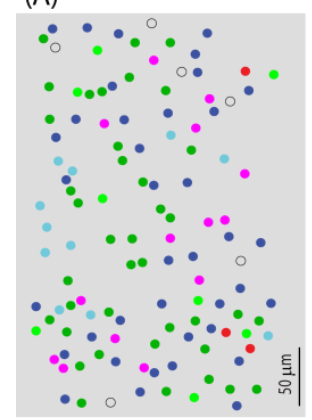

344

345

346

347

348

349

350
(B)

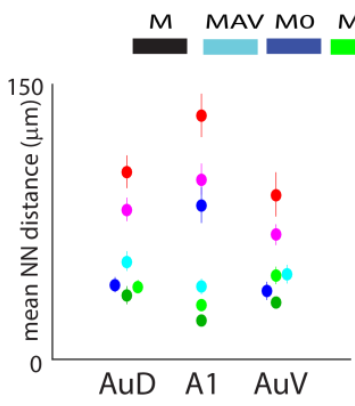

(C)

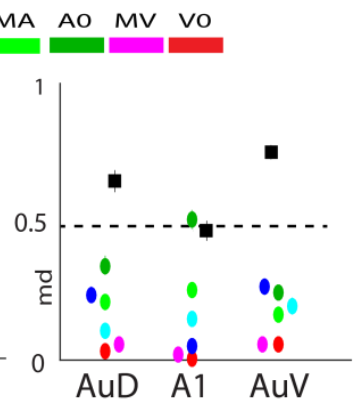

1

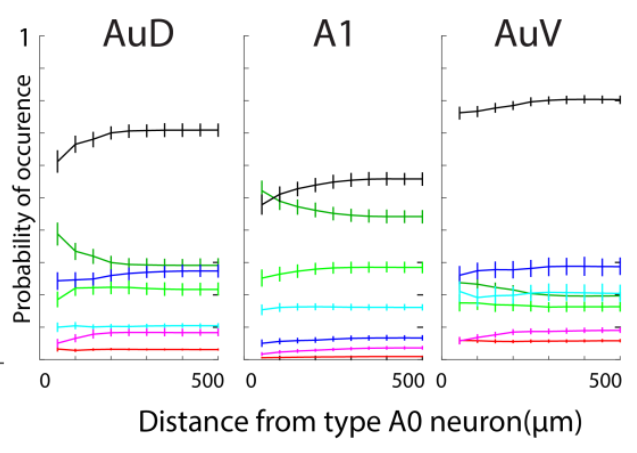

Figure 3. Spatial organization of different cell types w.r.t. type $\mathbf{A O}$ neurons in a local region. A, Example

recorded region with colour coded cell types. B, mean nearest neighbour (NN) distance between type

A0 neuron and the nearest neuron of a particular cell type (colour coded) in AuD, A1 and AuV regions of

the ACX. The coloured points are displaced to show the confidence intervals (also see table 2). C, mean

probability of occurrence of a certain cell type (colour coded) within 50um radius around type A0

neuron in AuD, $A 1$ and $A u V$ regions of the $A C X$. The coloured points are displaced to show the

confidence intervals (also see table 3)). D, Probability of occurrence of different cell types (colour coded) 
w.r.t type $A 0$ within a given radii (x axis). Black: type $M$ neurons, Cyan: type MAV, Blue: type M0, Light green: type MA, Dark green: type A0, Pink: type MV, Red: type V0 neurons.

\section{Layer 2/3 neurons in A1 respond primarily by hyperpolarization to visual stimulus}

$22 \%$ of responding neurons in $\mathrm{A} 1$ respond to visual stimulation (V0, MV, MAV, see Table 1, example ROI

in Fig. 4A\&B). Majority of these neurons in A1 respond by hyperpolarization to visual stimulus (68\%).

Some fraction of neurons in secondary auditory areas also respond by hyperpolarization but majority of them respond by excitation (Fig. 4A, lower panel). Response by excitation could be a result of direct or indirect excitatory drive from the visual cortex, while the response by hyperpolarization must involve inhibitory neurons. In agreement with the above statement, we found several neurons which responded by hyperpolarization to visual stimulus and by excitation to auditory stimulus (Fig. 4C). In order to look at the role of inhibitory neurons in the processing of cross modal information, we looked at a specific population of $A 1$ neurons, a subpopulation of type MAV, which respond by

364 hyperpolarization to visual stimulus and by excitation to auditory stimulus which are $44 \%$ of all visual

365 responding neurons in A1. For such a differential response to happen there has to be an inhibitory input

366 on these neurons that respond to visual stimulus and does not respond to auditory stimulus (Fig. 4C 367 upper panel). We looked for all the inhibitory neurons in the vicinity of these neurons which show such 368 a behaviour but we found only two inhibitory neurons that show such a behaviour which is not enough 369 to explain the observations.

370 Thus, it is clear that the PV+, SOM+ inhibitory neurons of Layer $2 / 3$ are not directly involved in carrying 371 out differential response to visual and auditory stimuli. Therefore, following possibilities exist that can 372 explain the response by hyperpolarization to visual stimuli and excitation to auditory stimuli. First, 
373 inhibition of layer $2 / 3$ neurons could be mediated by the inhibitory drive from Layer 1 , which like in

374 visual cortical areas has been shown to modulate responses by auditory stimulus (Ibrahim et al., 2016b;

375 Chou et al., 2020). Second, this hyperpolarization could also be mediated by the excitation of layer5/6

376 inhibitory neurons either directly or indirectly (via another excitatory neuron in the ACX) by excitatory

377 drive from visual cortex (Fig. 4C, lower panel). The axon terminals of these inhibitory neurons in layer

$3785 / 6$ can potentially hyperpolarize the excitatory neurons in Layer2/3 of A1(lurilli et al., 2012).

379 We recorded responses to visual and auditory stimulus from layer 5/6 of the ACX using a 4 by 4 array of 380 electrodes (data also published in the paper (Sharma and Bandyopadhyay, 2020)). We separated the 381 recorded neurons into inhibitory and excitatory neurons based on the spike shapes (Fig. 4E). We 382 calculated the spike width, i.e. full width-half maximum followed by a threshold at $0.25 \mathrm{~ms}$ to separate 383 the inhibitory from the excitatory neurons. We were able to identify a sub-population of units (48 units, $3848 \%$ of all responding units) that did not respond to auditory stimulus but responded to a visual stimulus $385\left(\mathrm{~V} 1 \mathrm{~A} 0_{\text {Ephys }}\right.$, Fig. 4D), out of which 33 were excitatory and 15 were inhibitory neurons. To make sure that 386 all our electrodes were in the ACX and not the nearby V2 area, which is expected to have plenty of such 387 inhibitory and excitatory neurons, we looked at the auditory responses of close by units on the 388 neighbouring electrodes (Fig. 4F). We found that most units responded robustly to the auditory stimulus 389 and of these, $\sim 73 \%$ did not respond to the visual stimulus. Based on the latency of peak response to 390 visual and auditory stimulus and the post-hoc Nissl staining of the sliced brain (Sharma and 391 Bandyopadhyay, 2020), we claim that all of our electrodes were in the ACX. Thus, we claim the existence 392 of inhibitory and excitatory neurons in layer $5 / 6$ of the ACX as potential direct and indirect source that may drive hyperpolarization of neurons in layer2/3. 
(A)

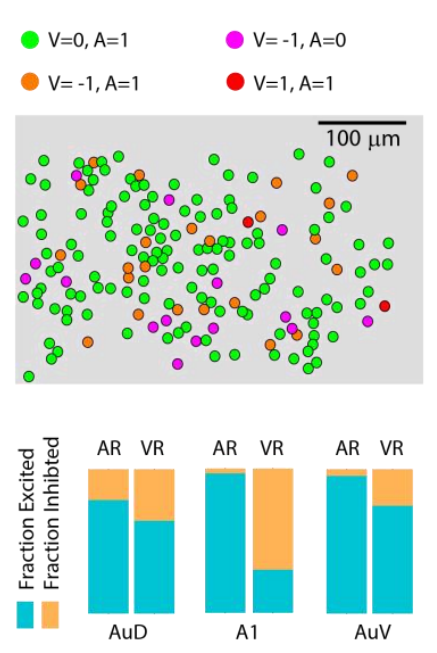

(D)

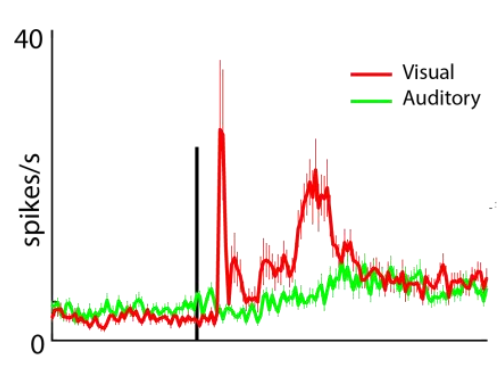

(B)
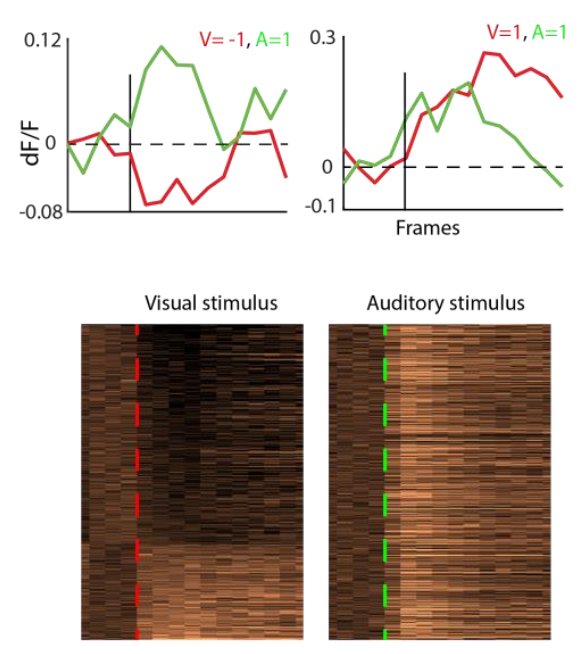

(E)

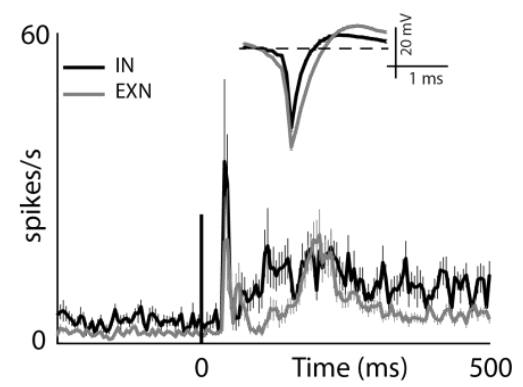

(C)
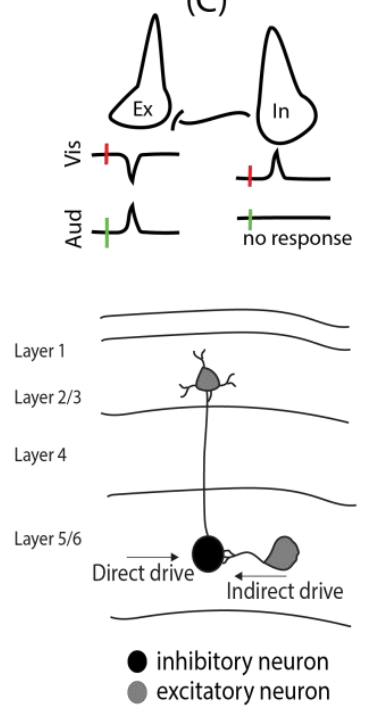

(F)

Figure 4. Neural underpinning of hyperpolarization behaviour of visual responding neurons in A1. A

396 (upper panel), Example ROI showing visual responding neurons in A1, colour coded by type of visual and auditory response. A (lower panel), Fraction of cells showing excitation and inhibition for auditory stimulus (AR) and visual stimulus (VR) in different regions of the ACX. Blue, fraction showing excitation; orange, fraction showing hyperpolarization. $\mathrm{B}$ (upper panel), Example traces of $\mathrm{dF} / \mathrm{F}$ signal from the ROI in A (upper panel). B (lower panel), response profiles of all visual responding cells in A1 for visual stimulus (left) and their response for auditory stimulus(right). C (upper panel), cartoon diagram explaining the possible cause of hyperpolarization responses. C(lower panel), hypothetical circuit model explaining the role of layer $5 / 6$ inhibitory neurons in causing hyperpolarization of layer $2 / 3$ neurons in 
405

406

407

408

409

response with SE of V1A0 inhibitory and excitatory neurons separated by color in layer 5/6 of ACX for visual stimulus with their respective spike shapes in the inset. $\mathbf{F}$, mean population response of neurons with SE located near to the above mentioned (in D) neurons showing response to auditory and visual stimulation.

\section{Effect of multisensory stimulation on the individual neurons and the network}

Given the large fraction of multisensory neurons within A1, we probed the nature of modulation during multisensory stimulus presentation. We took those neuron categories that showed significant modulation upon multisensory presentation as compared to unisensory presentation (MA, MAV, MO \& MV. Fig. 2, Table 1). We first compared the response properties of these neurons when presented with unisensory and multisensory stimuli and classified them as suppressed if the response to multisensory stimulus was significantly lower than the response to unisensory stimulus and enhanced if the response to multisensory stimulus was significantly greater than that to unisensory stimulus. The group of multisensory neurons whose responses were similar to both auditory and multisensory stimuli necessarily belonged to MAV (Methods, Classification of neurons).

Type MA neurons forms the greatest proportion of multisensory neurons in $A 1$, equivalent to type $A 0$ ( $M A=34 \%, A 0=34 \%)$ (Fig. 2B, middle and Table. 1). Almost all of the MA cells (98\%) showed significant suppression in responses upon multisensory presentation. Similar to A1, majority of MA neurons of secondary areas (AuD: 90\%, AuV: 93\%; Fig. 5A) also showed suppression upon multisensory presentation. Similarly for $M V$ neurons, majority of which also undergo suppression in A1 and in AuV, while in AuD, $14.6 \%$ neurons show enhancement of responses to multisensory stimulation. This percent can be explained by AuD's proximity with secondary visual areas. AuD previously has been shown to be 
427 multisensory, responding to both auditory and visual stimuli (Sharma and Bandyopadhyay, 2020).

428 Another point to notice here is that in the type MAV population, not all cells showed significant change

429 on multisensory presentation ( $85 \%$ ) and all those that did majority of them showed suppression (Fig.5A,

430 Table 1). Both type MV and type MA population showed suppression of responses on multisensory

431 presentation, regardless of the region specificity.

432 Neurons with similar stimulus preferences have higher noise correlations (Ecker et al., 2011) and change

433 in the correlations means change in the network involved. We attempted to decipher the network in

434 auditory and multisensory stimulation based on noise correlations. We calculated the correlation

435 coefficient on the mean subtracted trial data for each stimulus in the response window. We first looked

436 at our electrophysiology data, recorded from deep layer $2 / 3$ of the ACX, with auditory and multisensory

437 stimulus (Fig. 5B, left). We observed that during multisensory presentation, there was a decrease in

438 noise correlations among neurons (mean \pm se: $A ; 0.26 \pm 0.02$, MS; $0.15 \pm 0.02$, paired t-test $p<0.001$,

439 Fig.5B). We then computed the noise correlations during auditory and multisensory presentation from

440 Calcium signals of auditory responding cell types (A0, MA, MAV) and corroborated it with the

441 electrophysiology data. We found a similar trend from calcium signals as well, where there was a

442 significant decrease (95\% confidence with 1000 bootstraps) in noise correlations for multisensory

443 stimulus (mean \pm se $A=0.24 \pm 0.001, M S=0.19 \pm 0.001$, Fig. 5 C) .

444 Given higher noise correlations for auditory stimulus as compared to multisensory, we looked at the

445 contribution of auditory responding populations (A0, MA, MAV) to this effect (Fig.5C, right). We found

446 that $\mathrm{AO}$ (mean \pm se A: $0.27 \pm 0.002 \mathrm{MS}: 0.18 \pm 0.002$, Fig.5C,top) and MAV (A: $0.27 \pm 0.005$, MS: $0.19 \pm$

$447 \quad 0.005$, Fig.5C, bottom) cells have significantly higher noise correlations ( $95 \%$ confidence interval on 1000

448 bootstraps) during auditory stimulation and low for multisensory stimulation. While MA cells (A: $0.19 \pm$

4490.002, MS: $0.22 \pm 0.002$, Fig.5C, middle) show significantly higher noise correlations (95\% confidence on 

noise correlations for auditory stimulus compared to that of $\mathrm{AO}$ and MAV populations (Fig. 5C, right). Noise correlations can affect the neural population's capacity to discriminate between two stimuli without affecting the responses of the individual neurons(Downer et al., 2015). This suggests that $A 0$ population with very similar response profiles to auditory and multisensory stimulus relies on reduction

(A)

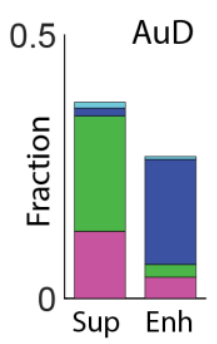

(B)
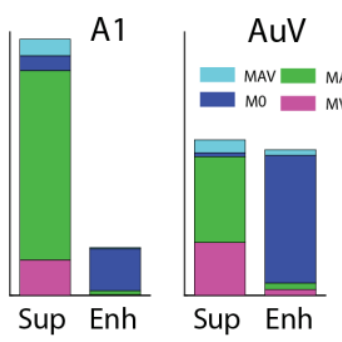

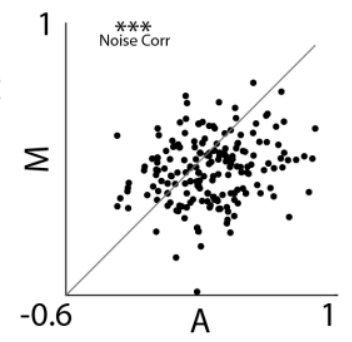

(C)

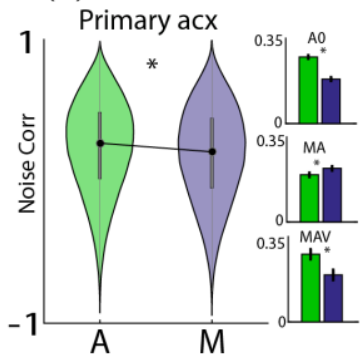

(D)

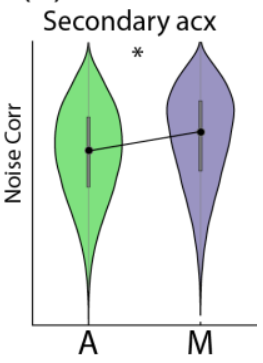

Figure 5. Multisensory modulation in the ACX. A, Multisensory neurons showing suppression and enhancement as a fraction of the total number of cells in that region, colour coded by type of neurons. between pairs of simultaneously recorded ACX neurons during electrophysiology experiments (*** $p$ - 
auditory (green) and multisensory (blue) presentation. The filled black dot shows the median of the

470 population and the vertical grey line shows the first and third quartiles. ${ }^{*}=$ significant based on $95 \%$

471 confidence interval from 1000 bootstraps. C right, bar plot of cell type wise mean noise correlations in

472 the A1. Top (A0), middle (MA), bottom (MAV). Green (auditory), blue (multisensory). ${ }^{*}=$ significant

473 based on $95 \%$ confidence interval from 1000 bootstraps. Error bars show the bootstrap confidence

474 interval. D, violin plot of population noise correlations in secondary ACX during auditory (green) and

475 multisensory (blue) presentation.

477 Discussion

478 In this study, we show that neurons in the ACX have complex multisensory architecture, comprising of

479 different neuron types that are affected by the presence of visual or multisensory stimulus in various

480 ways. Contrary to previous reports, approx. $20 \%$ neurons in layer $2 / 3$ of the ACX responded to visual

481 stimulation, including neurons that only responded to visual (type V0 and MV) stimulus and others

482 which responded to auditory stimulus as well (type MAV). This diversity of response types and thus of

483 neurons in the ACX was unknown previously. However, our classification of V0 and MV neurons is based

484 on the exercise that our auditory stimulus is a broadband noise. There could be a decrease in the

485 number of these cells if a pure tone were to be used as the auditory stimulus. Nevertheless, these

486 neurons would still be classified as visual responding multisensory cells.

487 When we look at the topography of the ACX at a greater resolution, we find neurons that respond to 488 visual stimulus, in close proximity to the neurons that respond exclusively to the presentation of 489 auditory stimulus (type A0). Type MV neurons which undergo modulation in presence of auditory 490 stimulus were prevalent and are present within 100um vicinity to auditory specific neurons (type A0). 
491 Multisensory stimulus do not modulate type A0 and type V0 neurons, they respond specifically to the

492 features of sound and the presence of visual and auditory stimulus respectively does not affect their

493 responses. These neurons may have a role in reliably transmitting information about auditory or visual

494 stimulus irrespective of other stimuli in the environment, as they have reliable responses to their

495 respective stimuli compared to other neuron types (Fig, 2C). Type MA and type MAV neurons are

496 present within 50um radii around type A0 neurons (Fig. 3C). Type MA neurons were as common in

497 primary ACX as were type A0 neurons, while they were greater in number in secondary areas. This close-

498 knit architecture is indicative of high multisensory influence on the processing of auditory information

499 and is capable of influencing auditory stimulus in multiple ways.

Response to visual stimulus differs between regions of the ACX. Greater numbers of neurons in

secondary areas respond by excitation to visual stimulation compared to neurons in primary auditory

502 area, where majority of neurons respond by hyperpolarization. This highlights a difference in processing

503 of visual stimulus in primary and secondary areas and the possible role of primary ACX in processing

504 visual information, which is to go silent. Silencing the primary sensory areas of non-sensory stimuli could

505 aid in processing the sensory stimuli (Dehner et al., 2004), yet keeping the secondary areas concerning

506 the non-sensory stimuli engaged to influence processing of the sensory stimuli. In addition, the local

$507 \mathrm{PV}+$ and SOM+ inhibitory neurons are not directly involved in causing these hyperpolarization responses

508 but the likely source of this hyperpolarization is in the layer 5/6 of the ACX(Kapfer et al., 2007; lurilli et

509 al., 2012). Layer $5 / 6$ of the ACX contains inhibitory neurons that do not respond to auditory stimulation

510 but to visual stimulation. This is a novel finding and puts forward another role of feedback from deeper

511 layers in influencing processing in the upper layers(Williamson and Polley, 2019). However, other studies

512 on the role of auditory stimulus in processing visual information, shows a role of layer 1 mediated

513 suppression in mice visual cortex (lurilli et al., 2012) and role of thalamus mediated suppression in 
monkeys (Chou et al., 2020) influencing processing in layer $2 / 3$ of the ACX. Similar processes can also

515 exist in mice affecting processing in the ACX by the visual stimulus.

Although few, but there were certain neurons of type MO which showed hyperpolarization on

517 multisensory stimulation (Fig. 2A, upper row and left column). Occurrence of such responses should

518 involve either summation of subthreshold inhibitory drive from neurons responding to auditory and

519 visual stimulus separately or inhibitory neurons of type M0 responding with excitation. We, in fact,

520 found inhibitory type MO neurons responding with excitation to multisensory stimulus. These neurons

521 could be a potential source of the hyperpolarization responses in type M0 population and a potential

522 explanation of widely observed suppression on multisensory stimulation (Fig. 5A). Another point of

523 difference between primary and secondary ACX stems from the noise correlation values during

524 multisensory stimulation. There is an increase in noise correlations among the neurons in the secondary

525 areas during multisensory stimulation while the correlations decrease in primary ACX. This decrease in

526 noise correlations means an increase in discriminability between stimuli(Downer et al., 2015). Thus,

527 primary ACX not only has a role in tone discriminability(O'sullivan et al., 2019) but also have network

528 able to discriminate between cross-modal stimuli. Our classification of auditory cortical regions into A1,

529 AuD and AuV is coarse(Kanold et al., 2014). Proper classification of different auditory cortical regions can

530 yield more specific information and functions of the fields of the ACX. In addition, lack of temporal

531 resolution in our recordings also limits our interpretations. Having access to greater temporal resolution

532 could yield better circuit dissection, information about possible anatomical pathways as reported in the

533 previous literature, and comparison of regions could be done in greater depth.

534 Several previous studies have shown the existence of visual inputs into the ACX, including 535 humans(Calvert et al., 1999), non-human primates(Kayser and Logothetis, 2007) ferrets(Bizley and King, 536 2008b), gerbils(Hackett and Schroeder, 2009), rats(Wallace et al., 2004a). While most of them deals with 
modulation of auditory cortical activity during multisensory presentation, a limited number of these

show spiking in the ACX by the visual stimulus in the deeper layers of the cortex (Morrill and Hasenstaub, 2018). Existence of these neurons in the superficial layers would allow tracking these neurons over days and establish the existence of these neurons more concretely. This will also allow us

541 to selectively look at the role of a given multisensory population type and its role in affecting the 542 processing of auditory and multisensory stimuli. Several previous studies have argued against the 543 unimodal nature of primary cortices(Wallace et al., 2004b; Ghazanfar and Schroeder, 2006; King and 544 Walker, 2012). This study is in concord with such studies and provides evidence for influence of 545 multisensory stimulus on the auditory information processing.

\section{References}

Atilgan H, Town SM, Wood KC, Jones GP, Maddox RK, Lee AKC, Bizley JK (2018) Integration of Visual Information in Auditory Cortex Promotes Auditory Scene Analysis through Multisensory Binding. Neuron 97:640-655.e4 Available at: http://www.ncbi.nlm.nih.gov/pubmed/29395914 [Accessed

Bizley JK, Jones GP, Town SM (2016) Where are multisensory signals combined for perceptual decisionmaking? Curr Opin Neurobiol 40:31-37 Available at: http://dx.doi.org/10.1016/j.conb.2016.06.003. 1242:24-36 Available at: nder.fcgi?artid=PMC4340571\%0Ahttp://dx.doi.org/10.1016/j.brainres.2008.02.087. 
Bizley JK, King AJ (2008b) Visual-auditory spatial processing in auditory cortical neurons. Brain Res.

Bizley JK, Nodal FR, Bajo VM, Nelken I, King AJ (2007) Physiological and anatomical evidence for multisensory interactions in auditory cortex. Cereb Cortex.

Budinger E, Heil P, Hess A, Scheich H (2006) Multisensory processing via early cortical stages: Connections of the primary auditory cortical field with other sensory systems. Neuroscience 143:1065-1083.

Bulkin DA, Groh JM (2012) Distribution of eye position information in the monkey inferior colliculus. J Neurophysiol.

Calvert GA, Brammer MJ, Bullmore ET, Campbell R, Iversen SD, David AS (1999) Response amplification in sensory-specific cortices during crossmodal binding. Neuroreport.

Campi KL, Bales KL, Grunewald R, Krubitzer L (2010) Connections of auditory and visual cortex in the prairie vole (microtus ochrogaster): Evidence for multisensory processing in primary sensory areas. Cereb Cortex.

Chamberland S, Yang HH, Pan MM, Evans SW, Guan S, Chavarha M, Yang Y, Salesse C, Wu H, Wu JC, Clandinin TR, Toth K, Lin MZ, St-Pierre F (2017) Fast two-photon imaging of subcellular voltage dynamics in neuronal tissue with genetically encoded indicators. Elife.

Chou XL, Fang Q, Yan L, Zhong W, Peng B, Li H, Wei J, Tao HW, Zhang LI (2020) Contextual and crossmodality modulation of auditory cortical processing through pulvinar mediated suppression. Elife.

Dehner LR, Keniston LP, Clemo HR, Meredith MA (2004) Cross-modal Circuitry between Auditory and Somatosensory Areas of the Cat Anterior Ectosylvian Sulcal Cortex: A "New" Inhibitory Form of Multisensory Convergence. Cereb Cortex. 
Deneux T, Kempf A, Bathellier B (2018) Context-dependent signaling of coincident auditory and visual events in primary visual cortex.

Downer JD, Niwa M, Sutter ML (2015) Task engagement selectively modulates neural correlations in primary auditory cortex. J Neurosci.

Ecker AS, Berens P, Tolias AS, Bethge M (2011) The effect of noise correlations in populations of diversely tuned neurons. J Neurosci.

Efron B, Tibshirani R (n.d.) An introduction to the bootstrap.

Ghazanfar AA (2005) Multisensory Integration of Dynamic Faces and Voices in Rhesus Monkey Auditory Cortex. J Neurosci 25:5004-5012 Available at: http://www.jneurosci.org/cgi/doi/10.1523/JNEUROSCI.0799-05.2005.

Ghazanfar AA (2009) The multisensory roles for auditory cortex in primate vocal communication. Hear Res.

Ghazanfar AA, Schroeder CE (2006) Is neocortex essentially multisensory? Trends Cogn Sci 10:278-285 Available at: https://www.sciencedirect.com/science/article/pii/S1364661306001045 [Accessed March 31, 2019].

Goldey GJ, Roumis DK, Glickfeld LL, Kerlin AM, Reid RC, Bonin V, Schafer DP, Andermann ML (2014) Removable cranial windows for long-term imaging in awake mice. Nat Protoc.

Hackett TA, Schroeder CE (2009) Multisensory integration in auditory and auditory-related areas of cortex. Hear Res.

Ibrahim LA, Mesik L, Ji X ying, Fang Q, Li H fu, Li Y tang, Zingg B, Zhang LI, Tao HW (2016a) Cross-Modality Sharpening of Visual Cortical Processing through Layer-1-Mediated Inhibition and Disinhibition. 
601

Neuron.

Ibrahim LA, Mesik L, Ji X ying, Fang Q, Li H fu, Li Y tang, Zingg B, Zhang LI, Tao HW (2016b) Cross-Modality Sharpening of Visual Cortical Processing through Layer-1-Mediated Inhibition and Disinhibition. Neuron 89:1031-1045 Available at: http://dx.doi.org/10.1016/j.neuron.2016.01.027.

lurilli G, Ghezzi D, Olcese U, Lassi G, Nazzaro C, Tonini R, Tucci V, Benfenati F, Medini P (2012) SoundDriven Synaptic Inhibition in Primary Visual Cortex. Neuron.

Kanold PO, Nelken I, Polley DB (2014) Local versus global scales of organization in auditory cortex. Trends Neurosci.

Kapfer C, Glickfeld LL, Atallah B V., Scanziani M (2007) Supralinear increase of recurrent inhibition during sparse activity in the somatosensory cortex. Nat Neurosci.

Kayser C, Logothetis NK (2007) Do early sensory cortices integrate cross-modal information? Brain Struct Funct.

Kayser C, Logothetis NK, Panzeri S (2010) Visual Enhancement of the Information Representation in Auditory Cortex. Curr Biol.

King AJ, Walker KMM (2012) Integrating information from different senses in the auditory cortex. Biol Cybern 106:617-625.

Knöpfel T, Sweeney Y, Radulescu Cl, Zabouri N, Doostdar N, Clopath C, Barnes SJ (2019) Audio-visual experience strengthens multisensory assemblies in adult mouse visual cortex. Nat Commun.

Koelewijn T, Bronkhorst A, Theeuwes J (2010) Attention and the multiple stages of multisensory integration: A review of audiovisual studies. Acta Psychol (Amst). 
621 Maddox RK, Atilgan H, Bizley JK, Lee AKC (2015) Auditory selective attention is enhanced by a task-

622 irrelevant temporally coherent visual stimulus in human listeners. Elife.

623 Meijer GT, Montijn JS, Pennartz CMA, Lansink CS (2017) Audiovisual Modulation in Mouse Primary

624 Visual Cortex Depends on Cross-Modal Stimulus Configuration and Congruency. J Neurosci

625 37:8783-8796 Available at: http://www.ncbi.nlm.nih.gov/pubmed/28821672 [Accessed March 12,

626 2019].

Morrill RJ, Hasenstaub AR (2018) Visual information present in infragranular layers of mouse auditory cortex. J Neurosci.

Murray MM, Thelen A, Thut G, Romei V, Martuzzi R, Matusz PJ (2016) The multisensory function of the human primary visual cortex. Neuropsychologia 83:161-169 Available at: http://dx.doi.org/10.1016/j.neuropsychologia.2015.08.011. eNeuro.

Oh SW et al. (2014) A mesoscale connectome of the mouse brain. Nature.

Romanski LM, Hwang J (2012) Timing of audiovisual inputs to the prefrontal cortex and multisensory integration. Neuroscience.

Sharma S, Bandyopadhyay S (2020) Differential Rapid Plasticity in Auditory and Visual Responses in the Primarily Multisensory Orbitofrontal Cortex. eNeuro 7.

Skipper JI, Van Wassenhove V, Nusbaum HC, Small SL (2007) Hearing lips and seeing voices: How cortical areas supporting speech production mediate audiovisual speech perception. Cereb Cortex. 
Acad Sci 101:2167-2172 Available at:

643

http://www.pnas.org/lookup/doi/10.1073/pnas.0305697101.

644 Wallace MT, Ramachandran R, Stein BE (2004b) A revised view of sensory cortical parcellation. Proc Natl

645 Acad Sci.

646

Wang Y, Celebrini S, Trotter Y, Barone P (2008) Visuo-auditory interactions in the primary visual cortex of the behaving monkey: Electrophysiological evidence. BMC Neurosci.

648 Werner-Reiss U, Kelly KA, Trause AS, Underhill AM, Groh JM (2003) Eye position affects activity in primary auditory cortex of primates. Curr Biol 13:554-562.

650 Williamson RS, Polley DB (2019) Parallel pathways for sound processing and functional connectivity among layer 5 and 6 auditory corticofugal neurons. Elife. 